\title{
Basin size evolution between dissipative and conservative limits
}

\author{
Paulo Cesar Rech, ${ }^{1,2, *}$ Marcus Werner Beims, ${ }^{2,3, \dagger}$ and Jason A. C. Gallas ${ }^{2,3,4, \$}$ \\ ${ }^{1}$ Departamento de Física, Universidade do Estado de Santa Catarina, 89223-100 Joinville, Brazil \\ ${ }^{2}$ Departamento de Física, Universidade Federal do Paraná, 81531-990 Curitiba, Brazil \\ ${ }^{3}$ Institut für Computerphysik, Universität Stuttgart, D-70569 Stuttgart, Germany \\ ${ }^{4}$ Instituto de Física, Universidade Federal do Rio Grande do Sul, 91501-970 Porto Alegre, Brazil
}

(Received 16 July 2004; revised manuscript received 7 October 2004)

Recent methods for stabilizing systems like, e.g., loss-modulated $\mathrm{CO}_{2}$ lasers, involve inducing controlled monostability via slow parameter modulations. However, such stabilization methods presuppose detailed knowledge of the structure and size of basins of attraction. In this paper, we numerically investigate basin size evolution when parameters are varied between dissipative and conservative limits. Basin volumes shrink fast as the conservative limit is approached, being well approximated by Gaussian profiles, independently of the period. Basin shrinkage and vanishing is due to the absence of bounded motions in the Hamiltonian limit. In addition, we find basin volume to remain essentially constant along a peculiar parameter path along which it is possible to recover the dissipation rate solely from metric properties of self-similar structures in phase-space.

DOI: XXXX

A characteristic feature of nonlinear systems is that they often exhibit more than one dynamic equilibrium state for the same value of model parameters. Some states may be chaotic while others are regular (periodic). The coexistence of several dynamic equilibrium states (attractors) was observed quite early in $Q$-switched $\mathrm{CO}_{2}$ lasers and called generalized multistability [1]. Several recent studies have shown multistability to be a property that may be profitably exploited in a variety of ways in lasers and other systems. For instance, multistability may be induced or suppressed by weak periodic perturbations [2-4], it is possible to control and limit the emergence of multistability [5-7], the phenomenon of noise-induced preference of attractors as well as the detection of multiple stable states and attractor hopping was also investigated [8]. Underlying all these widespread stabilization procedures is a need for detailed knowledge of the structure and extension of basins of attraction in phase-space. For instance, a riddled phase-space severely restricts any possibility of stabilization and is prone to catastrophic bifurcations from riddled to fractal basins [9]. This restriction is particularly severe in coupled systems $[10,11]$.

Here we report an investigation of basin size evolution when parameters are varied between dissipative and conservative limits. The model investigated is the standard example in the field $[3,7,10,12]$, the Hénon map $(x, y) \mapsto\left(a-x^{2}\right.$ $+b y, x)$, which affords ease of computation and clarity of presentation apart from well-modeling $\mathrm{CO}_{2}$ lasers in the limit of strong dissipation [12]. Of interest is to investigate how basin sizes change when moving between dissipative and conservative limits. As is known, while conservative systems are plagued by chaos for quite large sets of initial conditions, the dissipative limit with about the same complexity displays chaos only on relatively limited domains of the parameter

\footnotetext{
*E-mail address: rech@ fisica.ufpr.br

${ }^{\dagger}$ E-mail address: mbeims@ fisica.ufpr.br

${ }^{\ddagger}$ URL: http://www.ica1.uni-stuttgart.de/ jgallas
}

PACS number(s): 05.45.Gg, 05.45.Ac, 42.55.Lt

space $[13,14]$. The subtleties of the passage between conservative and dissipative limits have been discussed [15]. Exponential decay of basin size evolution was found recently for the Hénon map [16], along the characteristic path in parameter space where strange attractors disappear $[17,18]$.

Although questions related with basin volumes are of an elementary nature, their answers are nevertheless quite hard to come by: lacking adequate theoretical means to even es-
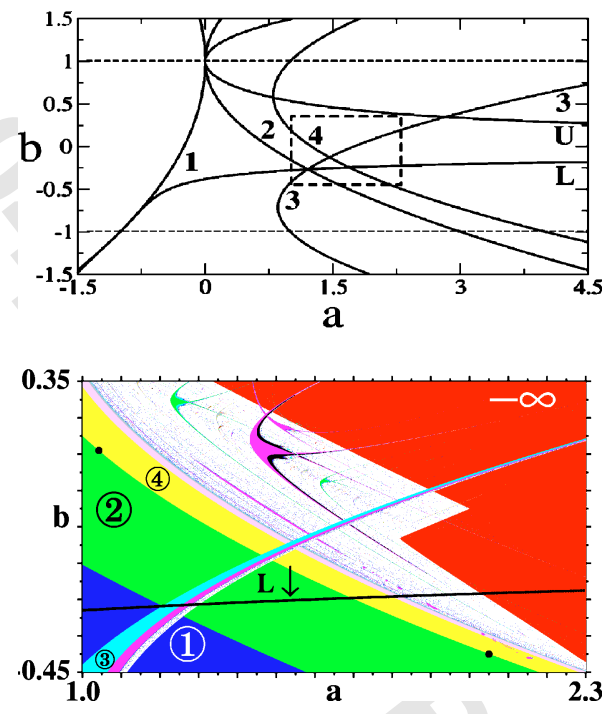

FIG. 1. (Color online) Top: Schematic view of boundaries between stability regions. Numbers label bifurcation loci: 1 marks the $0 \rightarrow 1$ saddle-node bifurcation, 2 marks the $1 \rightarrow 2$ bifurcation while 4 marks the $2 \rightarrow 4$ bifurcation. $U$ and $L$ refer to the upper and lower branches of the eigenvalue path (see text). The dashed rectangular box is shown magnified in the lower part of the figure. Bottom: Phase diagram showing the intricate alternation of stability domains underlying $L$ branch of the eigenvalue path $E(a, b)$. Different shadings denote different periods. Encircled numbers indicate the periodicity of the underlying domain. White represents chaos. The basin of unbounded attractors is indicated by $-\infty$. 

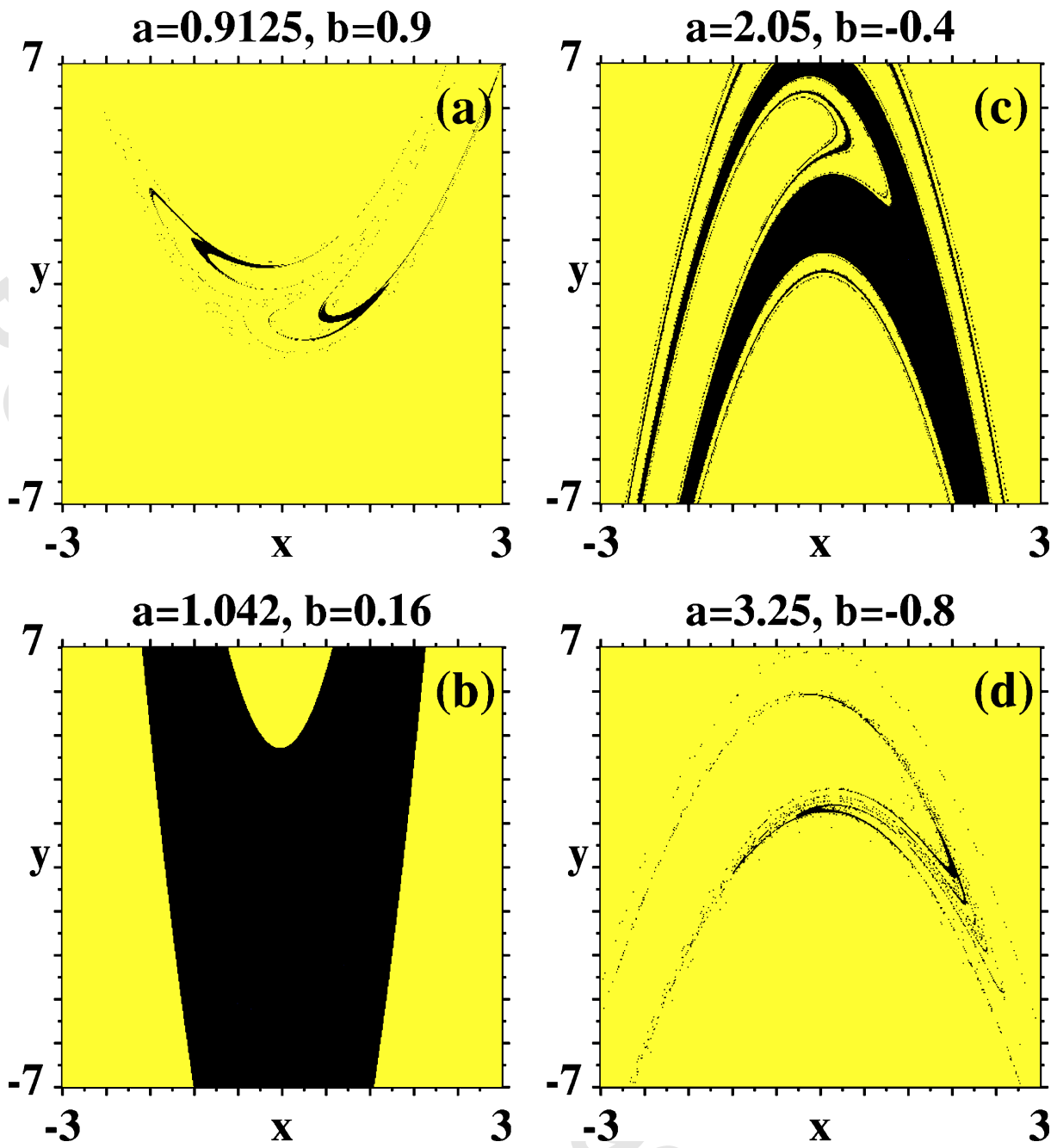

FIG. 2. (Color online) Illustrative basins computed for four different set of parameters along the $2 \rightarrow 4$ bifurcation line, defined by Eq. (3): (a) $(a, b)=(0.9125,0.9)$; (b) $(1.042,0.16)$; (c) $(2.05,-0.4)$; (d) $(3.25,-0.8)$. The grey shading indicates the basin of $-\infty$, black gives the basin of the periodic motion. The scale shown applies to all figures.

timate the answers, one needs to resort to intensive numerical computations, which we do here.

The exact description of the dynamics of a system which suffers a transition from conservative into dissipative is also very important in other contexts like, for example, energy transfer dynamics in system plus environment models [19], irreversibility in complex systems, charge and energy transfer in quantum molecular systems [20], and even in describing dissipation in quantum mechanics [21]. Presently, the key motivation for investigating basin evolution is the need for uncovering flexible and realistic maps to be used as discrete oscillators ruling local dynamics in lattices typically used in practical applications such as, for example, in simulations of aspects of ocean convection [22,23], particularly those connected with complex lattice topology and evolution [24-26].

Most of the time, parameter changes produce only minor modifications in the dynamical behavior. But while moving between dissipative and conservative limits one eventually crosses parameter paths like, for instance, stability or bifurcation loci, that are particularly interesting in several respects [17]. Here we concentrate on properties measured along a few of the simplest paths which are very convenient because they are all known analytically: the lines delimiting the boundaries of stability domains for low period oscillations and a very peculiar eigenvalue path [27] along which one may recover the dissipation rate by measuring the scaling of certain fractal fingers that appear in phase space.

Figure 1 illustrates the complex alternation of stability islands seen in a particularly crowded region of parameter space where we want to investigate basin size evolution. It was generated as described in Ref. [17] and concentrates on the physically meaningful strip $-1 \leqslant b \leqslant 1$. The wide white domain located roughly along the "main diagonal" of the figure represents parameters leading to stable chaotic solutions. The different shadings (tonalities) embedded in the white background represent stability islands of periodic attractors, different shadings denoting different periodicity. The large region on the upper right corner signals parameters leading mainly to unbounded solutions, i.e., to the attractor located at infinity (divergence).

Basin size evolution is studied along the parameter paths delimiting stability regions for trajectories of periods 1, 2, and 4. Such boundaries are the roots of the expressions 

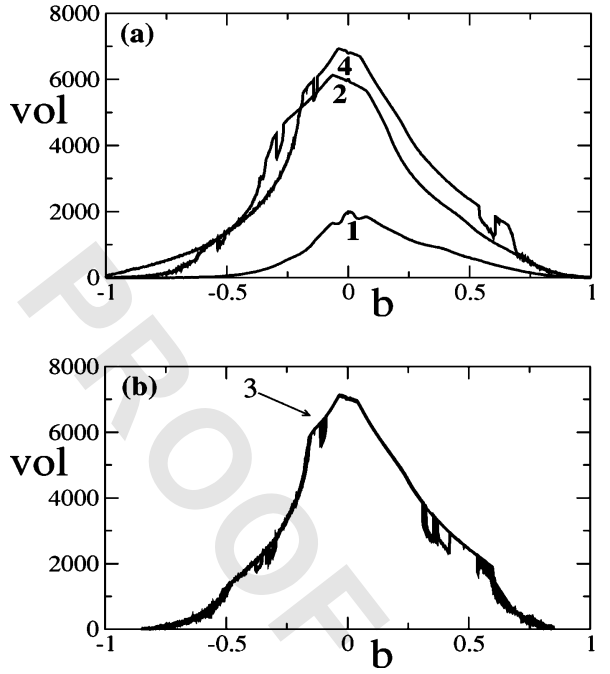

FIG. 3. (a) Basin volume for the three lowest periods of the 1 $\times 2^{n}$ cascade, as a function of the dissipation $b$. (b) Overlap of five curves, showing basin volume as a function of the dissipation $b$ for periods $8,16,32,64,128$, and 256 . The five curves overlap almost identically. The only noticeable differences involve intervals where multistability is present, e.g., the period-3 window indicated by 3 .

$$
\begin{gathered}
U(a, b) \equiv a+\frac{1}{4}(1-b)^{2}=0, \\
D(a, b) \equiv a-\frac{3}{4}\left(b^{2}-2 b+1\right)=0, \\
Q(a, b) \equiv a-\frac{1}{4}\left(5 b^{2}-6 b+5\right)=0 .
\end{gathered}
$$

The aforementioned eigenvalue path contains an upper branch $U$ and a lower branch $L$, which may be easily obtained by solving $E(a, b)=0$, where [27]

$$
E(a, b)=4 a b^{2}-\left[(b+1)^{4}-b^{2}\right](b-1)^{2} .
$$

For Eqs. (1)-(4), we determined the volume of basins of attraction for a total of $N=2000$ parameter values $P_{\ell}$ $\equiv\left(a_{\ell}, b_{\ell}\right), \ell=1,2, \ldots, N$ equally distributed along the bifurcation curves and located inside the strip $-1 \leqslant b \leqslant 1$. Figure 2 shows typical basin shape and volume for representative parameters along the $2 \rightarrow 4$ bifurcation locus. As seen, basins are quite small near the conservative limit $b=1$ and the limit $b=-1$. The figure also shows that basins may be fractal for certain parameters. The pair of black basins in Figs. 2(b) and 2(c) are computed for the parameters indicated by black dots in the lower part of Fig. 1. As evident from Figs. 2(a) and 2(d), basins are already quite small for $b=-0.8$ and $b=0.9$ although one is still far from the limits $|b|=1$. Basin volumes are maximal near $b \simeq 0$.

For each set of parameters $P_{\ell}$ we determined histograms of the periodicities observed in phase-space inside the window $-2.5 \leqslant x \leqslant 2.5$ and $-11 \leqslant y \leqslant 11$, discretized with a resolution of $100 \times 100$ points. This window contains the largest portion of "useful" initial conditions, namely those not in the basin of infinity.
Figure 3(a) displays the fraction of the $10^{4}$ initial conditions that do not diverge while moving from $b=-1$ to $b=1$ along three lines: the saddle-node $0 \rightarrow 1$ line (indicated by 1 ) and the $1 \rightarrow 2$ and $2 \rightarrow 4$ bifurcation lines. As seen, the volume increases with the unfolding of the bifurcation cascade. Furthermore, independently of the period, the volume tends to zero near $b=-1$ and $b=1$, the conservative (Hamiltonian) limit, attaining its maximum value for $b=0$, the limit where the map is noninvertible (nondiffeomorphic). Figure 3(a) also shows a few abrupt discontinuities, which simply indicate regions where there is multistability [1]. Of course, no discontinuities exist when all coexisting periods are added. We computed histograms using different discretizations and believe Fig. 3(a) to be accurate and representative. Figure 3(b) displays the overlap of five curves, showing basin volume as a function of the dissipation $b$ for periods $8,16,32$, 64,128 , and 256. The five curves overlap almost identically. Small differences appear only in intervals where multistability is present.

One discovers a number of interesting features when computing histograms along the branch $L$ of eigenvalue path $E(a, b)=0$. For instance, we have been able to numerically follow basin evolution for the $3 \times 2^{n}$ cascade during 6 bifurcations (up to period 96). During this rather long unfolding, the basin size remains essentially constant, reproducing the behavior described above for the $1 \times 2^{n}$ cascade [see Fig. 3 (b)]. The volume of the coexisting period- 2 basin also remains essentially constant in this interval of parameters. This shows that while intervals of stability in parameter space suffer strong compression as the bifurcation unfolds, basin volumes remain essentially unchanged. In other words, the immense difficulty to locate stable motions of high periodicity is linked only to the narrowness of parameter intervals, not to a decrease of basin volume.

A number of additional cascades are crossed when moving along $L$ up to $b=-0.26299$, for instance those starting with periods $9,15,21,27,33,45,63,75$, and 105. All these periodic cascades are separated by intervals where chaos prevails. Beyond $b=-0.26299$ one finds a sequence of attractors as summarized in Table I where, to save space, a few very small windows were omitted in some specific intervals. The last column in Table I gives the percentage of points not in the basin of $\infty$. As seen, even though there is a large variation of dynamical behaviors in this region, basin sizes remain essentially constant as before.

From Fig. 3 one sees that the basin volume decreases fast as $|b| \rightarrow 1$. To characterize this decrease we attempted fitting both a Gaussian and a log-normal to the volume distribution as a function of the $b$. However, since no significant improvement was found when using log normals, we only present here, in Fig. 4, the standard deviations $\sigma$ obtained when fitting Gaussians to basin volumes for motions with periods $1,2,4,8,16,32,64,128,256$, and chaos. The point at the right of the dotted line in the figure indicates a rough estimate of the standard deviation of the basin volume at the end of the periodic motions of the $1 \times 2^{n}$ cascade. The estimation of basin volumes at this extreme situation is much less certain than for periodic points. As is clear from Fig. 4, the standard deviation does not converge to any limit value, a fact that seems to agree well with reports [28] concerning 
TABLE I. Basin size variation relative to the basin of $\infty$. The interval corresponding to period 36 is very narrow. The first member of the $1 \times 2^{n}$ cascade lies outside the parameter interval considered in the table.

\begin{tabular}{c|c|c|c}
\hline \hline Attractor(s) & $b$ interval & $a$ interval & $\%$ \\
\hline $2,4, \ldots, 64$ & {$[-0.26298,-0.24060]$} & {$[1.30261,1.82574]$} & 35 \\
\hline Chaos & {$[-0.24059,-0.24012]$} & {$[1.82601,1.83878]$} & 35 \\
\hline 12,24 & {$[-0.24011,-0.24007]$} & {$[1.83906,1.84015]$} & 34 \\
\hline 36 & -0.24005 & 1.84069 & 34.5 \\
\hline Chaos & {$[-0.24004,-0.23978]$} & {$[1.84097,1.84807]$} & 35 \\
\hline $6,12, \ldots, 48$ & {$[-0.23977,-0.23946]$} & {$[1.84835,1.85685]$} & 35 \\
\hline Chaos & {$[-0.23945,-0.23918]$} & {$[1.85713,1.86457]$} & 34 \\
\hline 10 & {$[-0.23917,-0.23915]$} & {$[1.86485,1.86512]$} & 34 \\
\hline Chaos & {$[-0.23913,-0.23643]$} & {$[1.86595,1.94198]$} & 34 \\
\hline $7,14,28$ & {$[-0.23642,-0.23640]$} & {$[1.94227,1.94284]$} & 32 \\
\hline Chaos & {$[-0.23639,-0.23349]$} & {$[1.94313,2.02812]$} & 33 \\
\hline \hline
\end{tabular}

the basin size shrinkage factor with the period of the periodic attractors in connection with the unstable eigenvalue of the periodic orbit.

The Gaussian profiles reported here do not seem to be "universal." Exponentials were found [16] for the basin size evolution for the Hénon map along a path in the parameter space where the strange attractor disappears $[17,18]$. In contrast, here the parameter path is the boundary where the chaotic attractor is born. In addition, we offer an explanation for an interesting question posed by Feudel et al. [16], concerning how rare are chaotic basins in presence of multistability. Our figures show that not only are basins of chaotic attractors rare, but also rare are all basins related to periodic orbits. This property is not due to the multistability of the dynamical system, but arises from the open dynamics existent in the conservative limit of the Hénon map. In the Hamiltonian limit, there is no bounded motion: all orbits go to infinity and therefore basin sizes of all periodic or chaotic attractors simply do not exist anymore. In the dissipative limit most of

[1] F. T. Arecchi, R. Meucci, G. Puccioni, and J. Tredicce, Phys. Rev. Lett. 49, 1217 (1982).

[2] A. N. Pisarchik and R. Corbalán, Phys. Rev. E 59, 1669 (1999).

[3] A. N. Pisarchik and B. K. Goswami, Phys. Rev. Lett. 84, 1423 (2000).

[4] V. N. Chizhevsky, Phys. Rev. E 64, 036223 (2001).

[5] I. Triandaf and I. B. Schwartz, Phys. Rev. E 62, 3529 (2000).

[6] S. Gadaleta and G. Dangelmayr, Phys. Rev. E 63, 036217 (2001).

[7] A. N. Pisarchik, Phys. Rev. E 64, 046203 (2001).

[8] S. Kraut and U. Feudel, Phys. Rev. E 66, 015207(R) (2002).

[9] Y.-C. Lai, and V. Andrade, Phys. Rev. E 64, 056228 (2001).

[10] V. Astakhov, A. Shabunin, W. Uhm, and S. Kim, Phys. Rev. E 63, 056212 (2001).

[11] S. Yanchuk, K. R. Schneider, and L. Recke, Phys. Rev. E 69,

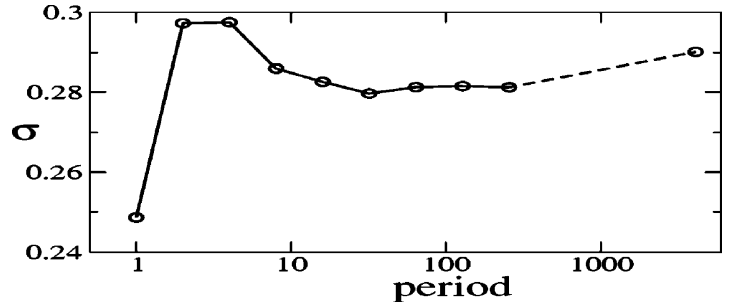

FIG. 4. The standard deviation of Gaussian fits to basin volume as a function of basin periodicity. See text. The rightmost point represents a rough estimate at the end of the $1 \times 2^{n}$ bifurcation cascade.

these points remain (due dissipation) confined to a finite region of the phase space.

In summary, basin sizes remain essentially constant as bifurcation cascades unfold, when the dissipation rate does not vary too much. On the other hand, there is a drastic drop in volume as one moves from the dissipative to the conservative (Hamiltonian) limit. As the conservative limit is approached, Gaussian profiles are found along stability boundaries between periodic orbits. Near the conservative limit, all basin sizes vanish for systems which have no bounded motions. These results are robust upon changes of the discretizations involved in their derivation and are insensitive to the rectangular domain of initial conditions used in phase-space to compute them. Knowledge of basin volume evolution in parameter space of a prototypical model map opens now the possibility of investigating aspects of the onset of synchronization and coherence in complex networks containing them as individual units and of answering a number of open questions [26].

This work was concluded during a visit of M.W.B. to the Institut für Computerphysik, Universität Stuttgart. He and J.G. are grateful to Professor Hans-Jürgen Herrmann for his kind invitation and hospitality. The authors also thank CAPES and CNPq, Brazil, for financial support. J.G. thanks support from the Sonderforschungsbereich 404 of the DPG, Germany.

056221 (2004)

[12] C. L. Pando, G. A.L. Luna Acosta, R. Meucci, and M. Ciofini, Phys. Lett. A 199, 191 (1995).

[13] A. J. Lichtenberg and M. A. Lieberman, Regular and Chaotic Dyamics (Springer, Berlin, 1992).

[14] Deterministic Chaos: An Introduction, edited by H. G. Schuster, 3rd ed. (Wiley, New York, 1998).

[15] P. V. Elyutin, Phys. Rev. E 69, 026205 (2004); , 69, 036207 (2004).

[16] U. Feudel and C. Grebogi, Phys. Rev. Lett. 91, 134102 (2003).

[17] J. A. C. Gallas, Phys. Rev. Lett. 70, 2714 (1993); A. Endler and J. A. C. Gallas, Physica A 344, 491 (2004).

[18] J. A. C. Gallas, C. Grebogi, and J. A. Yorke, Phys. Rev. Lett. 71, 1359 (1993).

[19] J. Rosa and M. W. Beims (unpublished).

[20] V. May and O. Kuhn, Charge and Energy Transfer Dynamics 
in Molecular Systems (Wiley-VCH, Berlin, 2000).

[21] A. H. Castro Neto and A. O. Caldeira, Phys. Rev. Lett. 67, 1960 (1991); U. Weiss, Quantum Dissipative Systems (World Scientific, Singapore, 1999).

[22] P. G. Lind, S. Titz, T. Kuhlbrodt, J. Corte-Real, J. Kurths, J. A. C. Gallas, and U. Feudel, Int. J. Bifurcation Chaos Appl. Sci. Eng. 14, 999 (2004).

[23] P. G. Lind, J. Corte-Real, and J. A. C. Gallas, Phys. Rev. E 69, 066206 (2004); 69, 026209 (2004).

[24] S. Strogatz, Sync: The Emerging Science of Spontaneous Or- der (Hyperion, New York, 2003).

[25] A.-L. Barabasi, Linked: The New Science of Networks (Perseus, New York, 2002).

[26] P. G. Lind, J. A. C. Gallas, and H. J. Herrmann, Phys. Rev. E 70, 056207 (2004); see also cond-mat/0407806.

[27] P. C. Rech, M. W. Beims, and J. A. C. Gallas, Europhys. Lett. 49, 702 (2000); Physica A 283, 273 (2000); Proceedings of the 11th IEEE Nonlinear Dynamics of Electronic Systems, edited by R. Stoop, Zurich, 2003, pp. 205-208.

[28] J. R. Miller and J. A. Yorke, Physica D 135, 195 (2000). 